International Mathematical Forum, Vol. 8, 2013, no. 35, 1709 - 1717 HIKARI Ltd, www.m-hikari.com

http://dx.doi.org/10.12988/imf.2013.39181

\title{
Dense Subsets in Semigroups ${ }^{1}$
}

\author{
Yuanlan Zhou \\ Department of Mathematics \\ Jiangxi Normal University, Nanchang \\ Jiangxi, 330022, P. R. China \\ ylzhou185@163.com \\ Yun Liu \\ Department of Mathematics \\ Yuxi Normal University, Yuxi \\ Yunnan 653100, P. R. China
}

Copyright (c) 2013 Yuanlan Zhou and Yun Liu. This is an open access article distributed under the Creative Commons Attribution License, which permits unrestricted use, distribution, and reproduction in any medium, provided the original work is properly cited.

\begin{abstract}
This article covers a theory of dense subsets in general semigroups, including basic algebraic properties of dense and disjunctive subsets, characterizations of dense subset preserving homomorphisms and some remarkable properties of dense elements.
\end{abstract}

\section{Introduction}

Let $A$ be a set, which we call an alphabet. The monoid $A^{*}$ is the free monoid on $A$. The set of nonempty word on $A$ is denoted by $A^{+}$, which is a semigroup and is called the free semigroup on $A$. The length $|w|$ of a word $w=a_{1} a_{2} \cdots a_{n}$ with $a_{i} \in A$ is the number $n$ of letters in $w$. We often denote by $w_{a}$ the number of occurrences of $a \in A$.

\footnotetext{
${ }^{1}$ This research is supported by the National Natural Science Foundation of China (10961014); the Science Foundation of the Education Department of Jiangxi Province.
} 
Any subset of a free monoid $A^{*}$ is called a language over $A$. There are many important classes of languages such as dense languages and disjunctive languages (that is, dense subsets and disjunctive subsets of free monoid, which we well define later) are investigated in literatures. In this paper, we well discuss such subsets in general semigroups.

Let $S$ be a semigroup, $L$ be any subset of $S$. For $x \in S$ we define the set of all contexts of $x$ with respect to $L$ by

$$
\operatorname{Cont}_{L}(x)=\left\{(u, v) \in S^{1} \times S^{1} \mid u x v \in L\right\} .
$$

The syntactic congruence of $L$, also called the (two sided) principal congruence of $L$, denoted by $P_{L}$ (or $\left.P_{L}(S)\right)$ is defined by $x P_{L} y$ if and only if $\operatorname{Cont}_{L}(x)=\operatorname{Cont}_{L}(y)$. That is:

$$
P_{L}=\left\{(x, y) \in S \times S \mid \forall u, v \in S^{1}(u x v \in L \leftrightarrow u y v \in L)\right\} .
$$

The left (resp. right) principal equivalences of $L$, denoted by $P_{L}^{(l)}$ (resp. $P_{L}^{(r)}$ ) are defined similarly by using left (resp. right) contexts of an element of $S$ with respect to $L$. For example,

$$
P_{L}^{(l)}=\left\{(x, y) \in S \times S \mid \forall u \in S^{1}(u x \in L \leftrightarrow u y \in L)\right\} .
$$

One verifies that $L$ is union of classes of $P_{L}$ (resp. $P_{L}^{(l)}, P_{L}^{(r)}$ ). In fact we have the following:

Proposition 1.1 (see [6]). $P_{L}$ (resp. $\left.P_{L}^{(l)}, P_{L}^{(r)}\right)$ is the largest congruence (resp. left congruence, right congruence) on $S$ for which $L$ is union of classes of $P_{L}\left(\operatorname{resp} . P_{L}^{(l)}, P_{L}^{(r)}\right)$.

Let $L$ be any subset of $S$. An element $x \in S$ is called completable (resp. left completable, right completable) in $L$ if

$$
\begin{gathered}
\exists u, v \in S^{1}(u x v \in L) \\
\text { (resp. } \left.\exists u \in S^{1}(u x \in L), \exists u \in S^{1}(x u \in L)\right) .
\end{gathered}
$$

$x$ is called bi-completable in $L$ if it is both left and right completable. An element which is not (left, right, bi-) completable in $L$ is called (left, right, $b i$-) incompletable. We call The subset $L$ dense if every elements of $S$ are completable in $L$. Otherwise we call it thin. Similarly we can define left (resp. right, bi-) dense and left (resp. right, bi-) thin. If the (left, right, bi-) dense subset $L$ is a singleton, the unique element of $L$ is called a (left, right, bi-) dense element. If the subsemigroup generated by $L$ is (left, right, bi-) dense then $L$ is called (left, right, bi-) complete, otherwise $L$ is called (left, right, bi-) incomplete. 
All the concepts above can be extend to monoids without modification. Let $S$ be a semigroup (resp. monoid), we define the syntactic semigroup (resp. monoid) of $L \subseteq S$ as $\operatorname{Syn}(L)=S / P_{L}$ and call the natural homomorphism from $S$ onto $\operatorname{Syn}(L)$ the syntactic homomorphism of $L$. We denote the $P_{L^{-}}$ class containing $x$ by $[x]_{L}$.

The subset $L$ of $S$ is called disjunctive (resp. left disjunctive, right dis-

junctive) if $P_{L}$ (resp. $P_{L}^{(l)}, P_{L}^{(r)}$ ) is the equality relation $1_{S}$. Notice that $L$ and $S \backslash L$ has the same principal (left, right) congruences, hence $L$ is (left, right) disjunctive in $S$ if and only if $S \backslash L$ is (left, right) disjunctive in $S$. This observation will be shown useful later.

If $S=A^{*}$ is the free monoid generated by an alphabet $A$, then the dense (resp. left dense, right dense, bi-dense) subsets of $S$ are called dense (resp. left dense, right dense, bi-dense) languages over $A$; and the disjunctive (resp. left disjunctive, right disjunctive) subsets of $S$ are usually called disjunctive (resp. left disjunctive, right disjunctive) languages over $A$.

The following proposition encourages us more or less to study the disjunctive subsets of general semigroups (especially the existence problem).

Proposition $1.2([6])$. A monoid $M$ is isomorphic to the syntactic monoid of a language $L$ over alphabet $A$ if and only if $M$ contains a disjunctive subset. Furthermore, $A$ can be chosen as a finite set if and only if $M$ is finitely generated.

\section{Basic Properties}

In the following discussions, we will only treat of dense and disjunctive subsets. Similar results are also hold, unless otherwise stated, for left (resp. right, bi-) dense subsets and left (resp. right) disjunctive subsets.

Proposition 2.1. Let $L \subseteq S$. Then $L$ is dense if and only if for any $x \in S$ there exist $u, v \in S$ such that $u x v \in S$.

Proposition 2.2. Let $L \subseteq S$. Then

(1) $L$ is dense in $S$ if and only if $L$ is dense in $S^{1}$.

(2) $L$ is disjunctive in $S$ if and only if $L$ is disjunctive in $S^{1}$.

The above proposition tells us that we can always consider dense and disjunctive properties in some monoids.

Proposition 2.3. If $L$ is dense subset of $S, L \subseteq L^{\prime} \subseteq S$ then $L^{\prime}$ is dense in $S$. 
Proposition 2.4. Let $L_{1}, L_{2}, \cdots, L_{n}$ be any nonempty subsets of $S$. Let $M=$ $L_{1} \cup L_{2} \cup \cdots \cup L_{n}, N=L_{1} L_{2} \cdots L_{n}$. Then

(1) $M$ is dense if and only if one of the $L_{i}(i=1, \cdots, n)$ is dense.

(2) If one of the $L_{i}(i=1, \cdots, n)$ is dense then $N$ is dense.

Remark 2.5. (1) In general, part (2) of this proposition is not hold for Left (resp. right, bi-) dense subsets. For example, $A^{*}$ is left dense language over $A$ but $A^{*} a$ is not, where $a$ is a letter in $A$.

(2) The converse of (2) in the above proposition is not true. For example, let $S=\{a, b, c\}$ be a semilattice such that $a b=c$. Let $L_{1}=\{a\}, L_{2}=\{b\}$. Then $L_{1}$ and $L_{2}$ are not dense but $L_{1} L_{2}=\{c\}$ is dense.

(3) If $S$ is a free monoid, then the converse of (2) in the above proposition is hold (see for example [10]).

Corollary 2.6. Finite semigroups always contain dense elements.

The finiteness condition in the above corollary is necessary. In fact, it is well know that free monoids have no finite dense subsets.

Proposition 2.7. If $S$ contains the zero element 0, then

(1) $L \subseteq S$ is dense if and only if $0 \in L$.

(2) The complements of dense (thin) subsets of $S$ are thin (dense).

(3) If $S$ contains disjunctive subsets, then $S$ contains both dense disjunctive subsets and thin disjunctive subsets.

(4) The concepts dense, left dense, right dense, bi-dense are all equivalent.

Proposition 2.8. If $S$ has no zero element, then the disjunctive subsets of $S$ are dense.

If $S=A^{*}$ is a free monoid, we have:

Proposition $2.9([8]) . L \subseteq A^{*}$ is dense if and only if it contains disjunctive subsets.

Proposition 2.10. Let $S$ and $T$ be any semigroups which have no zero elements, $X \times Y \subseteq S \times T$. Then:

(1) $X \times Y$ is dense in $S \times T$ if and only if $X$ and $Y$ are dense in $S$ and $T$ respectively.

(2) If $S$ and $T$ are monoids, then $X \times Y$ is disjunctive in $S \times T$ if and only if $X$ and $Y$ are disjunctive in $S$ and $T$ respectively.

Notice that the above proposition can be easily extend to the direct product of any collection of semigroups. 
Remark 2.11. The sufficiency of Item (2) in the above proposition is not hold in the semigroup without identity. For example, let $S=T=\{a, b\}$ be left zero semigroups and $X=Y=\{a\}$. Then $X$ and $Y$ are disjunctive in $S$ and $T$ respectively, but $X \times Y$ is not disjunctive in $S \times T$.

\section{Dense Subset Preserving Homomorphisms}

Let $S$ and $T$ be any semigroups. A homomorphism $\varphi$ from $S$ onto $T$ is said to recognize a subset $L$ of $S$ if $\varphi^{-1} \varphi(L)=L$.

Proposition 3.1. Let $S$ and $T$ be any semigroups, $\varphi$ be a homomorphism from $S$ onto $T, X \subseteq S$ and $Y \subseteq T$. Then

(1) If $X$ is dense then $\varphi(X)$ is also dense.

(2) If $Y$ is dense then $\varphi^{-1}(Y)$ is also dense.

In particular if $\varphi$ recognizes $X$, then $X$ is dense if and only if $\varphi(X)$ is dense.

Corollary 3.2. Let $S$ and $T$ be any semigroups, $\varphi$ be a homomorphism from $S$ onto $T, X \subseteq S$ and $Y \subseteq T$. Then

(1) If $X$ is complete then $\varphi(X)$ is also complete.

(2) If $Y$ is complete then $\varphi^{-1}(Y)$ is also complete.

In particular if $\varphi$ recognizes $X$, then $X$ is complete if and only if $\varphi(X)$ is complete.

In the formal language theory and the theory of codes, we often consider the homomorphisms which preserving some properties about languages. For example a homomorphism $\varphi: A^{*} \rightarrow B^{*}$ is called a code (resp. primitive word)-preserving homomorphism if $\varphi$ maps all codes (resp. primitive words) over $A$ to codes (resp. primitive words) over $B$ (see [9] or [10]). Let $S$ and $T$ be any semigroups, we can similarly define the dense (resp. complete) subset preserving homomorphism $\varphi: S \rightarrow T$. The above proposition tells us that any epimorphism is dense (resp. complete) subset preserving. For finitely generated free monoids, we can obtain a stronger property (see Theorem 3.9).

Proposition 3.3. Let $S$ and $T$ be any two semigroups (or monoids) generated by $A$ and $B$ respectively, $\varphi$ be a homomorphism from $S$ to $T$. Then

(1) $\varphi$ is dense subset preserving if and only if $\varphi(S)$ is dense in $T$.

(2) $\varphi$ is complete subset preserving if and only if $\varphi(A)$ is complete in $T$.

Corollary 3.4. Let $S$ and $T$ be any two semigroups and $\varphi$ be a homomorphism from $S$ to $T$. Then $\varphi$ is dense subset preserving if and only if $\varphi$ is complete subset preserving. 
Now we consider dense language preserving homomorphisms in free monoids. In the following discussion, we suppose $A$ and $B$ be any alphabets, $\varphi$ be a homomorphism from $A^{*}$ to $B^{*}$.

Lemma 3.5 (see [1]). Let $X$ be a thin code over $A$. Then $X$ is a maximal code if and only if $X$ is complete.

Lemma 3.6 (see $[\mathbf{1}, \mathbf{1 0}]) . \varphi$ is injective if and only if $\varphi(A)$ is a code over $B$ and $|\varphi(A)|=|A|$.

Proposition 3.7. If $\varphi$ is injective and $A$ is finite, then $\varphi$ is dense subset preserving if and only if $\varphi(A)$ is a maximal code.

The following example shows that the finiteness of $A$ is necessary.

Example 3.8. Let $B=\{a, b\}$ and $A$ be the restricted Dyck code over $B$. That is

$$
A=\left\{w \in B^{*} \mid w_{a}=w_{b} \text { and } u_{a}>u_{b} \text { for any nontrivial prefix } u \text { of } w\right\} .
$$

Let $\left.\varphi\right|_{A}$ be the embedding mapping. Since $A$ is a code over $B$, we have, by Lemma 3.6, $\varphi$ is injective. Since $A$ is dense in $B^{*}$, we have, by Proposition 3.3, $\varphi$ is dense subset preserving. But one can easily show that $A$ is not a maximal code over $B$.

Theorem 3.9. Let $A$ be a finite alphabet with $n>1$ letters. Then the endomorphism $\varphi$ of $A^{*}$ is dense subset preserving if and only if $\varphi$ is an automorphism.

Proof. We need only show the direct part.

Let $A=\left\{a_{1}, \cdots, a_{n}\right\}$ and $B=\varphi(A)=\left\{w_{1}, \cdots, w_{n}\right\}$. For any word $w \in A^{*}$, let $C(w)$ represent the content of $w$ (that is the set of letters occurring in $w$ ) and $c(w)=|C(w)|$. Let $m=\max \{|w| \mid w \in B\}$.

(1) If $c\left(w_{i}\right) \neq 1$ for some $i \in\{1, \cdots n\}$. Since $A$ is finite, there exists an $a_{i}$ such that $a_{i}^{+} \cap B=\emptyset$. Then $w=a_{i}^{2 m}$ is incompletable in $B^{*}=\varphi(A)^{*}=\varphi\left(A^{*}\right)$. That is $\varphi\left(A^{*}\right)$ is thin while $A^{*}$ is dense. Hence $\varphi$ can not preserve dense subsets, a contradiction.

(2) Hence $c\left(w_{i}\right)=1$ for all $i \in\{1, \cdots n\}$. That is $w_{i}=a_{k_{i}}^{s_{i}}$ with $k_{i} \in$ $\{1, \cdots n\}$ and $s_{i}>0$.

(i) If there exists an $s_{i}>1$. Then $a_{j} a_{k_{i}} a_{j},\left(k_{i} \neq j\right)$ is incompletable in $B^{*}=\varphi\left(A^{*}\right)$. A contradiction.

(ii) Hence $s_{i}=1$ for all $i$. That is $B \subseteq A$. If $w_{i}=w_{j}$ for some $i \neq j$. Since $A$ is finite, there exists an $a_{k} \notin B$. Then $a_{k}$ is incompletable in $B^{*}=\varphi\left(A^{*}\right)$. A contradiction. Hence $w_{i} \neq w_{j}$ for $i \neq j$. We have $B=\varphi(A)=A$. Hence $\varphi$ is an isomorphism. 
Corollary 3.10. If $A$ is a finite alphabet and $\varphi$ is a dense subset preserving homomorphism from $A^{*}$ to $B^{*}$. Then $|A| \geq|B|$. If $|A|=|B|$, then $\varphi$ is an isomorphism.

Disjunctive languages are generalized in some directions in the literature. $f$-disjunctive languages, $r f$-disjunctive languages, $n d$-disjunctive languages, $n i$-disjunctive languages, $g$-disjunctive languages, $q$-disjunctive languages and $q f$-disjunctive languages are studied in past decades (see $[2,3,4,7]$ ). We call all these languages the generalized disjunctive languages.

Corollary 3.11. Let $A$ be a finite alphabet with $n>1$ letters. Then the endomorphism $\varphi$ of $A^{*}$ is (generalized) disjunctive language preserving if and only if $\varphi$ is an automorphism.

\section{Dense Elements}

Now we cope with the (left, right, bi-) dense elements of a semigroup $S$. Let $J(S), L(S), R(S)$ and $H(S)$ represent the set of all dense, left dense, right dense, bi-dense elements respectively. Clearly, $H(S)=L(S) \cap R(S)$ and $L(S) \cup$ $R(S) \subseteq J(S)$. Moreover, we have:

Theorem 4.1. The following statements on any semigroup $S$ are equivalent.

(1) $J(S) \neq \emptyset$ (resp. $L(S) \neq \emptyset, R(S) \neq \emptyset, H(S) \neq \emptyset)$.

(2) $J(S)$ (resp. $L(S), R(S), H(S)$ ) is the minimum ideal (resp. left ideal, right ideal, quasi-ideal) of $S$.

(3) $S$ has the minimum ideal (resp. left ideal, right ideal, quasi-ideal).

(4) $J(S)$ (resp. $L(S), R(S), H(S)$ ) is the minimum $\mathcal{J}$ (resp. $\mathcal{L}, \mathcal{R}$, $\mathcal{H})$-class of $S$.

(5) $J(S)$ (resp. $L(S), R(S), H(S)$ ) is simple (resp. left simple, right simple, a group).

Corollary 4.2. Let $S$ be any semigroup. Then all elements of $S$ are dense (resp. left dense, right dense, bi-dense) if and only if $S$ is simple (resp. left simple, right simple, a group).

The following corollary will be shown useful in the formal language theory.

Corollary 4.3. Let $L$ be a language over $A$ and $\varphi: w \mapsto \bar{w}$ be the syntactic homomorphism of L. Denote

$$
\begin{aligned}
\bar{J} & =\left\{\bar{w} \in \operatorname{Syn}(L) \mid[w]_{L}=\varphi^{-1}(\bar{w}) \text { is a dense } P_{\left.L^{-} \text {-class }\right\}} .\right. \\
\bar{L} & =\left\{\bar{w} \in \operatorname{Syn}(L) \mid[w]_{L}=\varphi^{-1}(\bar{w}) \text { is a left dense } P_{L^{-}} \text {-class }\right\} \\
\bar{R} & =\left\{\bar{w} \in \operatorname{Syn}(L) \mid[w]_{L}=\varphi^{-1}(\bar{w}) \text { is a right dense } P_{L^{-} \text {class }}\right\} \\
\bar{H} & =\left\{\bar{w} \in \operatorname{Syn}(L) \mid[w]_{L}=\varphi^{-1}(\bar{w}) \text { is a bi-dense } P_{L^{-}} \text {-class }\right\} .
\end{aligned}
$$


Then clearly $\bar{H}=\bar{L} \cap \bar{R}$ and $\bar{L} \cup \bar{R} \subseteq \bar{J}$. Furthermore, the following statements are equivalent:

(1) $\bar{J} \neq \emptyset$ (resp. $\bar{L} \neq \emptyset, \bar{R} \neq \emptyset, \bar{H} \neq \emptyset)$.

(2) $\bar{J}$ (resp. $\bar{L}, \bar{R}, \bar{H}$ ) is the minimum ideal (resp. left ideal, right ideal, quasi-ideal) of $\operatorname{Syn}(L)$.

(3) Syn $(L)$ has the minimum ideal (resp. left ideal, right ideal, quasiideal).

(4) $\bar{J}$ (resp. $\bar{L}, \bar{R}, \bar{H}$ ) is the minimum $\mathcal{J}$ (resp. $\mathcal{L}, \mathcal{R}, \mathcal{H}$ ) class of $\operatorname{Syn}(L)$.

(5) $\bar{J}$ (resp. $\bar{L}, \bar{R}, \bar{H}$ ) is simple (resp. left simple, right simple, a group).

\section{References}

[1] Bestel, J. and Perrin, D., Theory of Codes, Academic Press, Orlando, 1985 .

[2] Guo, Y. Q., Shyr, H. J. and Thierrin, G., $q f$-disjunctive languages, Papers on Automata and Languages, Vol. 1 (1985), 1-28.

[3] Guo, Y. Q., Shyr, H. J. and Thierrin, G., f-Disjunctive Languages, International Journal of Computer Mathematics, Vol. 18 (1986), 219-237.

[4] Guo, Y. Q., Reis, C. M. and Thierrin, G., Relatively $f$-Disjunctive Languages, Semigroup Forum, Vol. 37 (1988), 289-299.

[5] Howie, J. M., An Introduction to Semigroup Theory, Academic Press, London, 1976.

[6] Lallement, G., Semigroups and Combinatorial Applications, Wiley Interscience, New York, 1979.

[7] Paradis, A. D., Shyr, H. J. and Thierrin, G., Quasi-Disjunctive Languages, Soochow Journal of Math, Vol. 3 (1982), 151-161.

[8] Reis, C. M. and Shyr, H. J., Some Properties of Disjunctive Languages on a Free Monoid, Information and Control, Vol. 37, No. 3 (1978), 334-344.

[9] Shyr, H. J. and Thierrin, G., Codes, Languages and MOL Schemes, R.A.I.R.O. Informatique Theorique / Theoretical Computer Science, Vol. 1, No. 4 (1977), 293-301. 
[10] Shyr, H. J., Free Monoids and Languages, Third Edition, Hon Min Book Company, Taichung, Taiwan, 2001.

Received: September 15, 2013 